\title{
A Receiver Architecture for Intra-Band Carrier Aggregation
}

\author{
Sy-Chyuan Hwu and Behzad Razavi \\ Electrical Engineering Department \\ University of California, Los Angeles, CA 90095
}

\begin{abstract}
A block downconversion receiver incorporates a digital image rejection technique to support multiple aggregated carriers by one receive path and one frequency synthesizer. A prototype consisting of a CMOS RF front end and an FPGA back end exhibits an image rejection ratio (IRR) of at least $70 \mathrm{~dB}$ across $2 \mathrm{GHz} \pm 25 \mathrm{MHz}$ and reconstructs a -76-dBm 64-QAM signal with an EVM of $-30 \mathrm{~dB}$ in the presence of another channel $40 \mathrm{~dB}$ higher.

Carrier aggregation is an attractive approach to increasing the bandwidth and hence the data rate in wireless communications. It is possible to place a single LO frequency mid-way between two carriers in a Weaver receiver so as to reconstruct simultaneously both signals [1]. However, this approach faces several issues: lack of frequency-dependent IRR calibration, the need for harmonic-rejection IF mixers, and the direct increase in analog complexity and power dissipation as the number of channels increases. The first issue is particularly important as even a $2 \%$ mismatch between the poles of two firstorder I and Q analog filters limits the usable bandwidth to $10 \%$ of the pole frequency if IRR must exceed $60 \mathrm{~dB}$.

This paper describes a "scalable" block downconversion receiver architecture that, by virtue of a background digital image calibration technique, can support multiple carriers while providing an IRR greater than $70 \mathrm{~dB}$. A new broadband lownoise amplifier (LNA) is also introduced.
\end{abstract}

Receiver Architecture The receiver architecture is shown in Fig. 1 along with spectra for a four-carrier example. The LO frequency is placed mid-way between the outermost carriers, thereby downconverting the block to an IF of no more than 35 $\mathrm{MHz}$ [1]. The quadrature IF signals are low-pass filtered, digitized, and applied to an image rejection module before complex downconversion to baseband. Of course, the two ADCs must digitize the IF components along with downconverted in-band blockers. Among the in-band blocker profiles in [2], that containing a $5-\mathrm{MHz}$ wide desired signal and a $40.5-\mathrm{dBc}$ blocker demands the widest ADC dynamic range (DR). Since a 64-QAM constellation dictates an SNR of about $24 \mathrm{~dB}$ for an acceptable BER, we conclude that the ADC must achieve a minimum DR of $64.5 \mathrm{~dB}$. Fortunately, recent work on ADCs has reported a 14-bit, 80-MHz converter that achieves an SNR of $71 \mathrm{~dB}$ and an SFDR of $80 \mathrm{~dB}$ at the Nyquist rate while consuming $31 \mathrm{~mW}$ [3]. If running at $80 \mathrm{MHz}$, this ADC oversamples the desired signal by a factor of 8 , achieving a DR of roughly $71 \mathrm{~dB}+10 \log 8 \approx 80 \mathrm{~dB}$, providing ample margin for the above scenario.

Illustrated in Fig. 2, our proposed algorithm is based on two principles. (1) With analog gain and phase mismatches of $\epsilon$ and $\theta$, respectively, we can simply multiply, in the frequency domain, the I component by a complex number $\alpha=$ $(1+\epsilon) \cos \theta+j(1+\epsilon) \sin \theta$ so as to remove the mismatches. (2) If two overlapping channels at IF carry a power of $P_{A}$ and $P_{B}$, then $|I|^{2},|Q|^{2}$, and the inner product, $I \cdot Q$, yield the values shown in Fig. 2, from which we can derive $\operatorname{Re}\{\alpha\}$ and $\operatorname{Im}\{\alpha\}$. Remarkably, since $\operatorname{Im}\{\alpha\}$ and $\operatorname{Re}\{\alpha\}$ can be calculated for each FFT bin, the frequency-dependent mismatches are corrected with a fine frequency resolution.

The operations required in the mismatch estimator are performed by bit-serial arithmetic using only adders and registers [4] because the computation of $\alpha$ can be as slow as temperature- and supply-induced drifts in $\epsilon$ and $\theta$. The phases of $\omega_{I F}$ in the downconverter are produced by numericallycontrolled oscillators, i.e., an accumulator followed by a lookup table, yielding harmonic-free mixing. The downconverter in Fig. 2 is repeated according to the number of intra-band carriers.

Receiver Front End Fig. 3 shows the implementation of the front end, designed for the LTE range of $700 \mathrm{MHz}$ to 2700 MHz. We propose a broadband LNA with active feedback so as to obtain a low noise figure (NF), acceptable input matching, and single-ended to differential conversion.

The circuit is designed such that, with the load presented by the mixers and the TIAs, the voltage gain of $\mathrm{Inv}_{2}$ is about unity and $R_{i n}=50 \Omega$. The LNA noise figure is $1.76 \mathrm{~dB}$.

Operating with low supply voltages, this LNA stands in contract to the noise-cancelling topology in [5], which does not cancel the noise of the input CG device if it drives balanced mixers and TIAs (and employs unequal drain resistors).

In order to establish a well-defined bias current for the inverters in the LNA, a servo loop adjusts the PMOS body voltage of a replica inverter, $\operatorname{Inv}_{\mathrm{rep}}$, so as to force $V_{1}$ equal to $V_{2}$, thus driving the bias current of $\operatorname{Inv}_{\text {rep }}$ toward $I_{\mathrm{REF} 1}$. This method obviates the need for placing a bias current source in series with the source of the inverter transistors, allowing a greater voltage headroom and hence higher linearity.

Experimental Results The receiver front end in Fig. 1 has been fabricated in TSMC 45-nm digital CMOS technology and tested with off-the-shelf ADCs and an FPGA back end. Shown in Fig. 4, the active die area is $450 \mu \mathrm{m} \times 350 \mu \mathrm{m}$. Tested at 2 $\mathrm{GHz}$, the prototype dissipates $15 \mathrm{~mW}$.

Shown in Fig. 5, the measured NF varies from $3.65 \mathrm{~dB}$ to $3.85 \mathrm{~dB}$, and the IRR exceeds $70 \mathrm{~dB}$ across the IF bandwidth. Figure 6 presents the measured performance for two carriers. As a first test, a tone is placed in Channel 1 and a modulated signal in Channel 2. We observe that the image of Channel 2 is reduced by more than $70 \mathrm{~dB}$ after $\alpha$ in Fig. 2 settles (in 1 $\mathrm{ms}$ ) to its correct value. In the next test, a QPSK or 64-QAM signal resides in Channel 1 and another modulated signal 40 $\mathrm{dB}$ higher in Channel 2. The measured constellations in Fig. 
6 demonstrate that the image due to Channel 2 is greatly suppressed, yielding an EVM of $-30 \mathrm{~dB}$ for the 64-QAM signal.

The complexity and power of the digital back end have been conservatively estimated from the FPGA implementation. The FFT and IFFT blocks, the mismatch estimator logic, and the downconverters require about $95 \mathrm{k}$ gates, which, for a gate density of $400 \mathrm{k} / \mathrm{mm}^{2}$ in $45-\mathrm{nm}$ technology, translates to $0.24 \mathrm{~mm}^{2}$. With a clock frequency of $70 \mathrm{MHz}$, the total power of the logic is estimated to be $10 \mathrm{~mW}$.

Table I summarizes the measured performance and compares it with other state-of-the-art receivers. (Only [1] supports carrier aggregation.)

Acknowledgements Research supported by Realtek Semiconductor. The authors thank the TSMC University Shuttle Program for chip fabrication.

\section{References}

[1] L. Sundstrom et al., ISSCC Dig., pp. 336-337, Feb. 2013.

[2] 3GPP TS 36.101 V11.5.0 (2013-07).

[3] R. Kapusta et al., IEEE JSSC, pp. 3059-3066, Dec. 2013.

[4] P.B. Denyer and D.Renshaw, VLSI signal processing: a bit-serial approach, Addison-Wesley, 1985.

[5] F. Bruccoleri, et al., IEEE JSSC, pp. 275-282, Feb. 2004.

[6] W. Riyan, et al., IEEE RFIT, pp. 85-88, 2011.

[7] H. Xie, et al., IEEE TMTT, pp. 1390-1396, May. 2012.

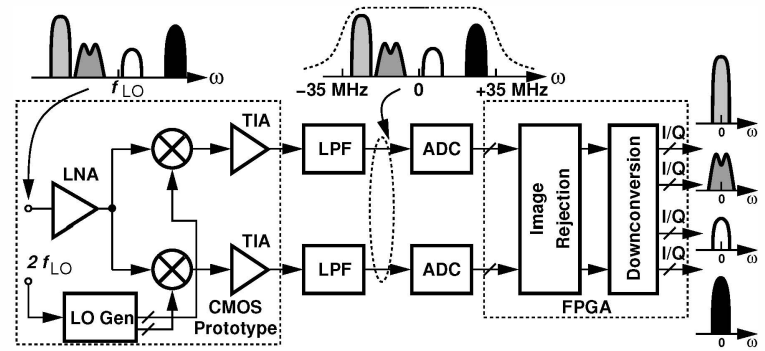

Fig. 1. Proposed receiver architecture.

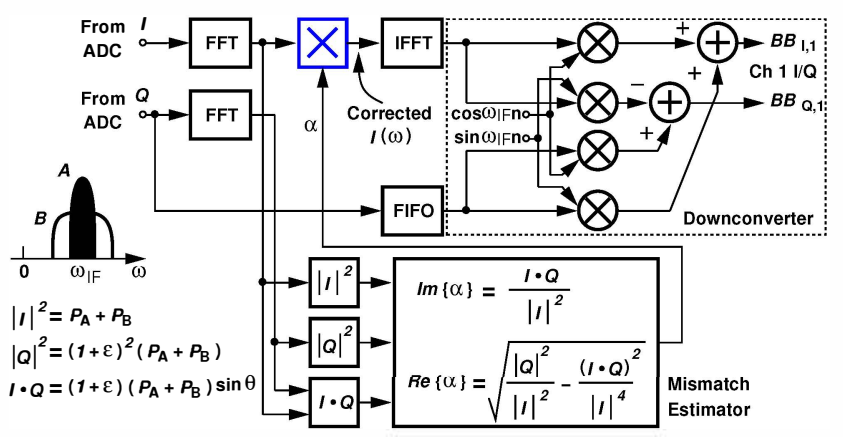

Fig. 2. Proposed image rejection and downconverter implementation.
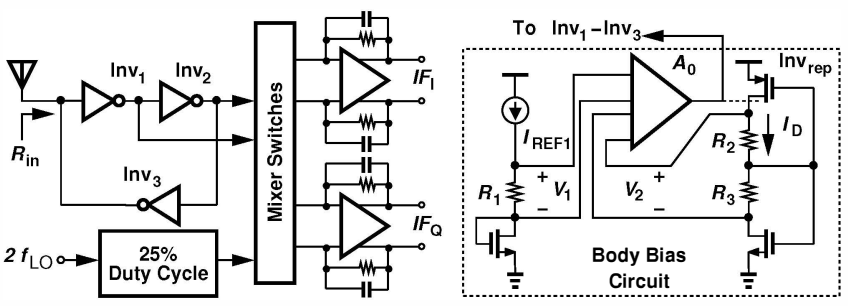

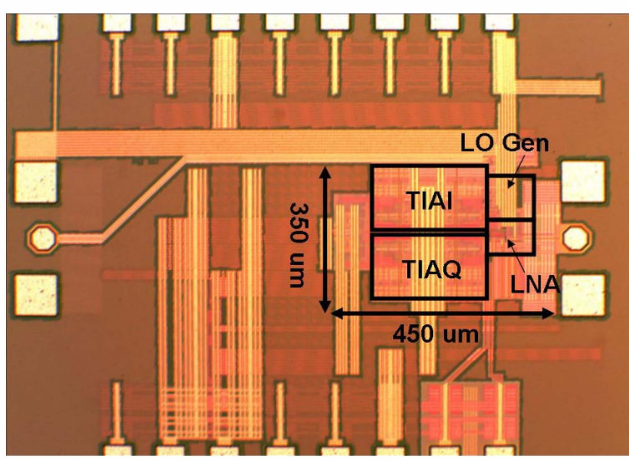

Fig. 4. Die photograph of receiver front end.
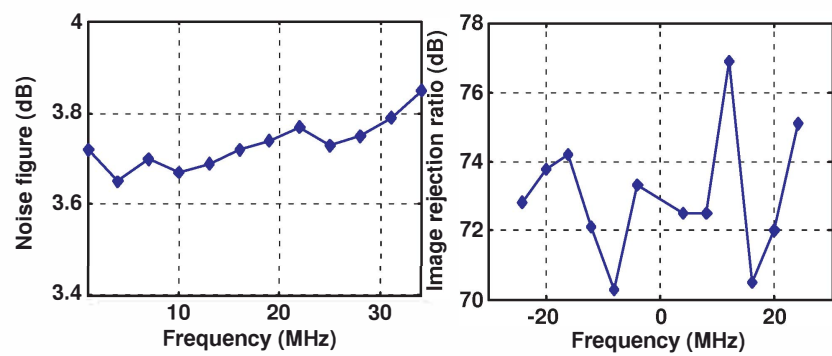

Fig. 5. Measured NF and image rejection ratio.
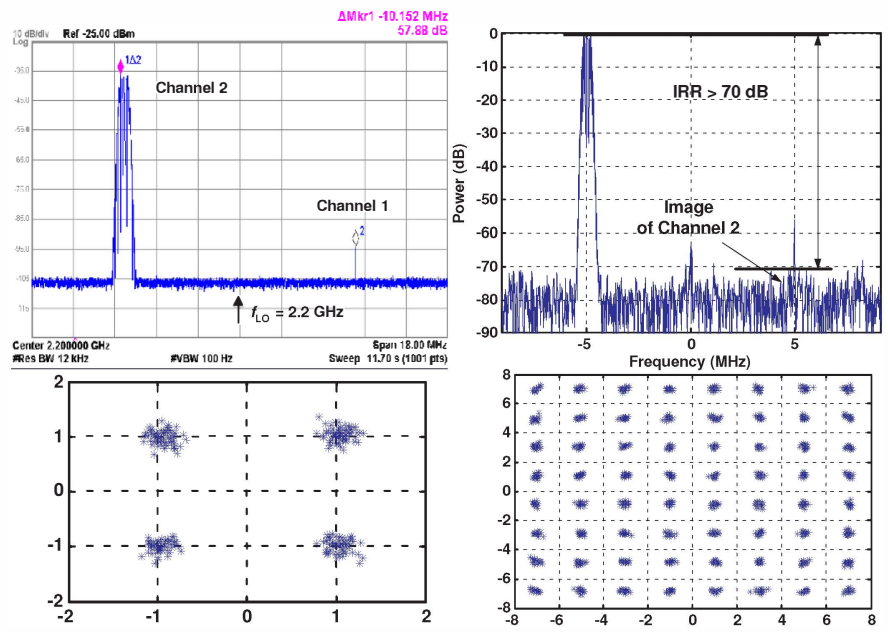

Fig. 6. Measured complex baseband spectrum and constellations. TABLE1: Performance Summary

\begin{tabular}{|c|c|c|c|c|}
\hline & This work & {$[1]$} & {$[6]$} & {$[7]$} \\
\hline Standard & LTE & LTE & LTE & LTEMCDMA \\
\hline Architecture & $\begin{array}{c}\text { Block } \\
\text { Down. }\end{array}$ & $\begin{array}{c}\text { Double } \\
\text { Conv. }\end{array}$ & Direct Conv. & Direct Conv. \\
\hline $\begin{array}{c}\text { No. of Intra- } \\
\text { Band Carriers }\end{array}$ & $>2$ & 2 & 1 & 1 \\
\hline Tech. / Supply & $45 \mathrm{~nm} / 1 \mathrm{~V}$ & $\begin{array}{c}65 \mathrm{~nm} / \\
1.45 \mathrm{~V}\end{array}$ & $130 \mathrm{~nm} / 1.2 \mathrm{~V}$ & $90 \mathrm{~nm} / \mathrm{NA}$ \\
\hline Power & $15 \mathrm{~mW}^{(1)}$ & $68 \mathrm{~mW}^{(2)}$ & $48 \mathrm{~mW}^{(3)}$ & $\mathrm{NA}$ \\
\hline IIP2 (duplex) & $>40 \mathrm{dBm}$ & $>58 \mathrm{dBm}$ & $60 \mathrm{dBm}$ & $>60 \mathrm{dBm}$ \\
\hline IIP3 (half-duplex) & $2.75 \mathrm{dBm}$ & $2.4 \mathrm{dBm}$ & $-7 \mathrm{dBm}$ & $2 \mathrm{dBm}$ \\
\hline IIP2 (in-band) & $31 \mathrm{dBm}$ & $27 \mathrm{dBm}$ & $\mathrm{NA}$ & $\mathrm{NA}$ \\
\hline IIP3 (in-band) & $-13.7 \mathrm{dBm}$ & $-15 \mathrm{dBm}$ & $\mathrm{NA}$ & $-8 \mathrm{dBm}$ \\
\hline DSB NF & $3.8 \mathrm{~dB}$ & $4.5 \mathrm{~dB}$ & $3.1 \mathrm{~dB}$ & $3 \mathrm{~dB}$ \\
\hline Voltage Gain & $37 \mathrm{~dB}$ & $45 \mathrm{~dB}$ & $45 \mathrm{~dB}$ & $60 \mathrm{~dB}$ \\
\hline IRR & $>70 \mathrm{~dB}$ & $>55 \mathrm{~dB}$ & $\mathrm{NA}$ & $\mathrm{NA}$ \\
\hline
\end{tabular}

(1) LNA + Mixers + TIAs + LO gen.

(2) Estimated from ISSCC Visual Supplement for LNA + RF mixers + IF mixers, excluding the power of all IF and baseband filters.

(3) LNA + Mixers + TIAs + LO gen.

Fig. 3. Implementation of receiver front end. 